\title{
JOHN SURFACES ASSOCIATED WITH A CLASS OF HARMONIC MAPPINGS
}

\author{
Willy Sierra \\ Universidad del Cauca, Departamento de Matemáticas \\ Popayán, Colombia; wsierra@unicauca.edu.co
}

\begin{abstract}
We study some criteria for the image of the unit disk under the canonical lift $\tilde{f}$ of a harmonic mapping $f$ to be a John surface and a linearly connected surface. Growth results of the metric of the minimal surface associated to $\tilde{f}$ are obtained. Some our results generalize known ones in geometric functions theory.
\end{abstract}

\section{Introduction}

If $f$ is an analytic and locally univalent function in a domain $\Omega$, we define the Schwarzian derivative of $f$ by

$$
S_{f}=\left(\frac{f^{\prime \prime}}{f^{\prime}}\right)^{\prime}-\frac{1}{2}\left(\frac{f^{\prime \prime}}{f^{\prime}}\right)^{2} .
$$

A central result, which relates the growth of the Schwarzian derivative with the univalence of $f$ was proven by Nehari in [13]. He proved that a function $f$ analytic and locally univalent in $\mathbf{D}:=\{z:|z|<1\}$ is univalent if

$$
\left|S_{f}(z)\right| \leq 2 p(|z|)
$$

where $p:(-1,1) \rightarrow \mathbf{R}^{+}$satisfies the following conditions: $p$ is a continuous and even function, $\left(1-t^{2}\right)^{2} p(t)$ is decreasing in $(0,1)$, and the differential equation $u^{\prime \prime}+p u=0$ has no nontrivial solutions with more than one zero in $(-1,1)$. A function $p$ satisfying these conditions is called a Nehari's function and the class $N$ of functions which satisfy the univalence criteria (1) with $p(t)=\left(1-t^{2}\right)^{-2}$, class of Nehari. In another direction, Ahlfors and Weill [2] and more recently, Gehring and Pommerenke [15] studied criteria in terms of size of $\left|S_{f}\right|$ for $f(\mathbf{D})$ to be a quasidisk. Other geometric and analytic properties of functions in the class $N$ were studied in [9] and [10]. In particular, in [9] the authors showed that

$$
\left|\frac{f^{\prime \prime}(z)}{f^{\prime}(z)}\right| \leq 2 \mu \frac{|z|}{1-|z|^{2}}, \quad 0<\mu \leq 1
$$

holds if $f$ satisfies (1) with $p(t)=\mu\left(1-t^{2}\right)^{-2}$ and $f^{\prime \prime}(0)=0$. In [10] it is shown that if $f \in N_{0}:=\left\{f \in N: f^{\prime \prime}(0)=0\right\}$, then $f(\mathbf{D})$ is a John domain if and only if

$$
\limsup _{|z| \rightarrow 1}\left(1-|z|^{2}\right)\left|\frac{f^{\prime \prime}}{f^{\prime}}(z)\right|<2 .
$$

doi:10.5186/aasfm.2013.3844

2010 Mathematics Subject Classification: Primary 30C55; Secondary 31A05, 53A10, 30C45.

Key words: Schwarzian derivative, John surface, harmonic mapping, minimal surface.

The author wishes to thank the University of Cauca for providing time for this work through research project VRI ID 3357. 
The main purpose of this paper is to establish sufficient conditions for image of the unit disk under a lifting of a planar harmonic mapping to be John surface (whose definition is similar to the standard definition of John domain in $\mathbf{R}^{n}$ ). Some our results generalize classical results of Ahlfors-Weill and Gehering-Pommerenke mentioned before. Also we obtain an analogue to (2) and (3) and growth estimates for the metric of the minimal surface associated to harmonic mapping.

A function $f=u+i v$ from a domain $\Omega$ in the $x y$-plane to $\mathbf{C}$ is harmonic if $\Delta u=\Delta v=0$, where $\Delta$ is the Laplace operator. If $\Omega$ is simply connected, there are analytic functions $h, g$ such that $f=h+\bar{g}$, and this representation is unique up to an additive constant. If $0 \in \Omega$, we will assume without loss of generality the unique representation $f=h+\bar{g}$ with $g(0)=0$. If $f=h+\bar{g}$ is a harmonic mapping in $\mathbf{D}$ whose dilatation $w_{f}:=\frac{g^{\prime}}{h^{\prime}}$ is the square of a meromorphic function $q$, then $f$ lifts locally into a regular minimal surface $\Sigma$ expressed by conformal parameters. According to the Weierstrass-Enneper formulas, the Cartesian coordinates $\left(x_{1}, x_{2}, x_{3}\right)$ of the surface are given by

$$
x_{1}=\operatorname{Re}\{f(z)\}, \quad x_{2}=\operatorname{Im}\{f(z)\}, \quad x_{3}=2 \operatorname{Im}\left\{\int_{z_{0}}^{z} h^{\prime}(\zeta) q(\zeta) d \zeta\right\},
$$

$z_{0} \in \Omega$. From now on, we write $\tilde{f}(z)$ for $\left(x_{1}(z), x_{2}(z), x_{3}(z)\right)$. The conformal factor of the first fundamental form is given by $\lambda=\left|h^{\prime}\right|+\left|g^{\prime}\right|$ and the Gaussian curvature at a point $\tilde{f}(z)$ on the surface by $K=-\lambda^{-2} \Delta(\log \lambda)$. In our case, this formula for $K$ reduces to

$$
K=-\frac{4\left|q^{\prime}\right|^{2}}{\left|h^{\prime}\right|^{2}\left(1+|q|^{2}\right)^{4}}
$$

For every such harmonic mappings with $\lambda(z) \neq 0$ for all $z \in \mathbf{D}$, in [3] is defined the Schwarzian derivative of $f$ by

$$
\mathcal{S} f=2\left(\partial_{z z}(\log \lambda)-\left(\partial_{z} \log \lambda\right)^{2}\right) .
$$

An important property of $\mathcal{S} f$ to be used later is the equality

$$
\mathcal{S}(f \circ \varphi)=(\mathcal{S} f \circ \varphi)\left(\varphi^{\prime}\right)^{2}+S_{\varphi},
$$

which holds for $\varphi$ analytic and locally univalent in the unit disk and $f$ harmonic with the properties specified above. It will be important in the proof of Lemma 1 other expression for $\mathcal{S} f$ obtained in [3]; namely,

$$
\mathcal{S} f=S_{h}+\frac{2 \bar{q}}{1+|q|^{2}}\left(q^{\prime \prime}-\frac{q^{\prime} h^{\prime \prime}}{h^{\prime}}\right)-4\left(\frac{q^{\prime} \bar{q}}{1+|q|^{2}}\right)^{2} .
$$

On the other hand, a generalization of the univalence criteria of Nehari (1) is proven in [4]. In that article is shown that, if $f=h+\bar{g}$ is harmonic in $\mathbf{D}$, its dilatation is the square of some meromorphic function, and

$$
|\mathcal{S} f(z)|+\lambda^{2}(z)|K(z)| \leq 2 p(|z|),
$$

then the lifting $\tilde{f}$ is univalent in $\mathbf{D}$ and admits a continuous extension to $\overline{\mathbf{D}}$. Moreover, if the strict inequality holds in (8), then the extension of $\tilde{f}$ to $\overline{\mathbf{D}}$ is univalent.

From now on, by a harmonic function $f$ we mean a harmonic function in $\mathbf{D}$ whose dilatation is the square of some meromorphic function. We will denote by $\lambda_{f}$ 
the associated density to $f$ or, simply $\lambda$ when no confusion can arise. Note that if $\varphi$ is analytic from $\mathbf{D}$ into $\mathbf{D}$ and $f$ is harmonic, then $f \circ \varphi$ is harmonic and

$$
\lambda_{f \circ \varphi}=\left(\lambda_{f} \circ \varphi\right)\left|\varphi^{\prime}\right| .
$$

We will denote by $N H^{\mu}$ the family of harmonic functions $f$ satisfying

$$
|\mathcal{S} f(z)|+\lambda^{2}(z)|K(z)| \leq \frac{2 \mu}{\left(1-|z|^{2}\right)^{2}}, \quad 0<\mu \leq 1 .
$$

$N H_{0}^{\mu}$ will denote the family of functions $f \in N H^{\mu}$ with $\partial_{z} \lambda(0)=0$. We will write $N H$ and $N H_{0}$ instead of $N H^{1}$ and $N H_{0}^{1}$, respectively. As in the analytic case, it is easy to see that if $\varphi \in \operatorname{Aut}(\mathbf{D})$ and $f \in N H^{\mu}$, then $f \circ \varphi \in N H^{\mu}$. This follows directly from (6) and (9).

Further background is discussed in Section 2. Below, we summarize some of our results.

Theorem 1. If $f \in N H_{0}^{\mu}, 0<\mu<1$, then

a.) $\Sigma=\tilde{f}(\mathbf{D})$ is a John surface with center $\tilde{f}(0)=p$ and the curves $\gamma_{\xi}(t)=\tilde{f}(t \xi)$, $0 \leq t \leq 1$, are John curves;

b.) there are $\alpha \in(0,1)$ and $K>0$ (depending only on $\mu$ ) such that

$$
\frac{\left(1-\rho^{2}\right) \lambda(\rho \xi)}{\left(1-r^{2}\right) \lambda(r \xi)} \leq K\left(\frac{1-\rho}{1-r}\right)^{\alpha}
$$

where $\xi \in \mathbf{T}$ and $0 \leq r<\rho<1$;

c.) there are $\alpha \in(0,1)$ and $K_{1}>0$ (depending only on $\mu$ ) such that for all $z=r e^{i t} \in \mathbf{D}$,

$$
\frac{\left(1-|\zeta|^{2}\right) \lambda(\zeta)}{\left(1-|z|^{2}\right) \lambda(z)} \leq K_{1}\left(\frac{1-|\zeta|}{1-|z|}\right)^{\alpha}, \quad \zeta \in B(z)
$$

where $B\left(r e^{i t}\right):=\left\{\rho e^{i \theta}: r \leq \rho \leq 1,|\theta-t| \leq \pi(1-r)\right\}$.

Theorem 2. Suppose $f$ is a harmonic mapping which satisfies

$$
|\mathcal{S} f(z)|+\lambda^{2}(z)|K(z)| \leq 2 p(|z|)
$$

and let $c=\lim _{t \rightarrow 1}\left(1-t^{2}\right)^{2} p(t)$. If $c<1$ and $\tilde{f}$ is bounded, then $\tilde{f}(\mathbf{D})$ is a John surface with center at $p=\tilde{f}(0)$.

Remark 1. Let $p$ be a Nehari function and $c=\lim _{t \rightarrow 1}\left(1-t^{2}\right)^{2} p(t)$. By an argument of comparison of solutions of differential equations one can show that $c \leq 1$ (see [5]). In that paper also is proven that $c=1$ implies $p(t)=\left(1-t^{2}\right)^{-2}$. The function $L(z)=\frac{1}{2} \log \frac{1+z}{1-z}$ shows that the conclusion of the before theorem is false if $c=1$.

Theorem 3. Let $0<\mu<1$. If $f=h+\bar{g} \in N H^{\mu}$ and $\tilde{f}$ is bounded, then $\tilde{f}(\mathbf{D})$ is a linearly connected surface.

A surface $S \subset \mathbf{R}^{n}$ is said to be a $b$-surface of John, with $b>1$, if there is a point $p \in S$, which is called center of the John surface, such that every point $q \in S$ can be joined to $p$ by a rectifiable curve $\gamma \subset S$ from $p$ to $q$ satisfying the following condition:

$$
\ell(\gamma(y, q)) \leq b d(y, \partial S) \text { for all } y \in \gamma
$$


where $\gamma(y, q)$ denotes the subarc of $\gamma$ from $y$ to $q$ and $\ell(\gamma(y, q))$ its length. Here and subsequently, $d(q, \partial S)$ stands for the extended real number

$$
d(q, \partial S):=\sup \left\{r \geq 0: B_{S}(q, r) \subset S\right\},
$$

where $B_{S}(q, r)$ is the ball in $S$ centered at $q$ with radius $r$. In this case we will say that $\gamma$ is a John curve.

Also, a slight modification to the standard definition of linearly connected domain in the plane, leads us to the following definition, which we will use in this paper: a surface $S \subset \mathbf{R}^{n}$ is said to be linearly connected, if there is $M>0$ such that for all $a, b \in S$, there is a curve $\Gamma \subset S$ with endpoints $a$ and $b$ satisfying the property

$$
\operatorname{diam}(\Gamma) \leq M|a-b|
$$

Here, the diameter is understood in the metric of the surface.

\section{The Schwarzian derivative of Ahlfors}

In this paper it plays a prominent role the concept of Schwarzian derivative of a curve in the space, which was introduced by Ahlfors in [1]. The Schwarzian derivative of a regular curve $\varphi:(a, b) \rightarrow \mathbf{R}^{n}$ of class $C^{3}$, also called Ahlfors' Schwarzian, is defined by

$$
S_{1} \varphi=\frac{\left\langle\varphi^{\prime}, \varphi^{\prime \prime \prime}\right\rangle}{\left|\varphi^{\prime}\right|^{2}}-3 \frac{\left\langle\varphi^{\prime}, \varphi^{\prime \prime}\right\rangle^{2}}{\left|\varphi^{\prime}\right|^{4}}+\frac{3}{2} \frac{\left|\varphi^{\prime \prime}\right|^{2}}{\left|\varphi^{\prime}\right|^{2}} .
$$

A key property of the Ahlfors' Schwarzian is its invariance under post-composition with Möbius transformation of $\mathbf{R}^{n} . S_{1} \varphi$ also satisfies the real analogous of (6). More precisely, if $x:(c, d) \rightarrow(a, b)$ is of class $C^{3}$ with $x^{\prime}(t) \neq 0$ for all $t \in(c, d)$, then

$$
S_{1}(\varphi \circ x)(t)=S_{1} \varphi(x(t)) x^{\prime}(t)^{2}+S_{1} x(t)
$$

where

$$
S x:=S_{1} x=\left(\frac{x^{\prime \prime}}{x^{\prime}}\right)^{\prime}-\frac{1}{2}\left(\frac{x^{\prime \prime}}{x^{\prime}}\right)^{2} .
$$

For our purpose, it will be useful an expression for $S_{1}$ obtained in [8], namely,

$$
S_{1} \varphi=\left(\frac{v^{\prime}}{v}\right)^{\prime}-\frac{1}{2}\left(\frac{v^{\prime}}{v}\right)^{2}+\frac{1}{2} v^{2} k^{2}=S s+\frac{1}{2} v^{2} k^{2},
$$

where $v$ is the velocity of $\varphi, k$ its curvature, and $s$ its arc length. We collect in the below theorems some of the results proven in [8]:

Theorem 4. Let $P$ be a continuous function on $(-1,1)$ such that no nontrivial solution $u$ of the differential equation $u^{\prime \prime}+P u=0$ has more that one zero. Let $\varphi:(-1,1) \rightarrow \mathbf{R}^{n} \cup\{\infty\}$ be a regular curve of class $C^{3}$. If $S_{1} \varphi(x) \leq 2 P(x)$ on $(-1,1)$, then $\varphi$ is injective.

Theorem 5. Let $\varphi$ and $P$ be as before theorem. Suppose moreover than $P$ is even and $\varphi$ satisfies $\varphi(0)=0,\left|\varphi^{\prime}(0)\right|=1, \varphi^{\prime \prime}(0)=0$, and $S_{1} \varphi(x) \leq 2 P(x)$. Let $u$ be the solution of the equation $u^{\prime \prime}+P u=0$ with the initial conditions $u(0)=1$ and $u^{\prime}(0)=0$ and we define the function $F(x)=\int_{0}^{x} \frac{d t}{u^{2}(t)}$. Then
a.) $\left|\varphi^{\prime}(x)\right| \leq F^{\prime}(|x|)$ on $(-1,1)$ and $\varphi$ has a spherically continuous extension to $[-1,1]$
b.) if $F(1)<\infty$, then $\varphi$ is injective on $[-1,1]$ and $\varphi$ has finite length;
c.) if $F(1)=\infty$, then either $\varphi$ is injective on $[-1,1]$ or, up to rotation, $\varphi=F$. 


\section{Estimates for the metric of the surface}

We start this section with a lemma which generalizes (2) to the class $N H_{0}$.

Lemma 1. Let $0<\mu \leq 1$ and $f \in N H_{0}^{\mu}$. Then

$$
\left|\frac{\partial \log \lambda}{\partial z}(z)\right| \leq \frac{\mu|z|}{1-|z|^{2}}
$$

for all $z \in \mathbf{D}$.

Proof. Consider first the case when $\mu=1$. Since $N H_{0}$ is invariant under composition with rotations of the unit disk, it is sufficient to prove the lemma for $0<z<1$.

From the equality $\lambda=\left|h^{\prime}\right|+\left|g^{\prime}\right|=\left|h^{\prime}\right|\left(1+|q|^{2}\right)$ we deduce that

$$
\partial_{z} \log \lambda=\frac{1}{2} \frac{h^{\prime \prime}}{h^{\prime}}+\frac{q^{\prime} \bar{q}}{1+|q|^{2}}
$$

and therefore, if $y(t)=\partial_{z} \log \lambda(t)$, then

$$
\begin{aligned}
y^{\prime} & =\frac{1}{2}\left(\frac{h^{\prime \prime}}{h^{\prime}}\right)^{\prime}+q^{\prime \prime} \frac{\bar{q}}{1+|q|^{2}}+q^{\prime} \frac{(1+q \bar{q}) \overline{q^{\prime}}-\bar{q}\left(q \overline{q^{\prime}}+\bar{q} q^{\prime}\right)}{\left(1+|q|^{2}\right)^{2}} \\
& =\frac{1}{2} S_{h}+\frac{1}{4}\left(\frac{h^{\prime \prime}}{h^{\prime}}\right)^{2}+q^{\prime \prime} \frac{\bar{q}}{1+|q|^{2}}+\frac{\left|q^{\prime}\right|^{2}}{\left(1+|q|^{2}\right)^{2}}-\left(\frac{\bar{q} q^{\prime}}{1+|q|^{2}}\right)^{2} .
\end{aligned}
$$

It follows, by using (7), that

$$
y^{\prime}=\left(\frac{1}{2} \frac{h^{\prime \prime}}{h^{\prime}}+\frac{q^{\prime} \bar{q}}{1+|q|^{2}}\right)^{2}+\frac{1}{2} \mathcal{S} f+\frac{\left|q^{\prime}\right|^{2}}{\left(1+|q|^{2}\right)^{2}},
$$

whence in view of (4) and (14),

$$
y^{\prime}=y^{2}+\left(\frac{1}{2} \mathcal{S} f-\frac{1}{4} \lambda^{2} K\right) .
$$

Hence, by (8) and (15), we conclude that $\varphi(t)=|y(t)|$ satisfies

$$
\varphi^{\prime} \leq \varphi^{2}+\frac{1}{\left(1-t^{2}\right)^{2}}
$$

Note that by definition of $N H_{0}, \partial_{z} \lambda(0)=0$ and therefore $\varphi(0)=0$. Consider now the initial value problem

$$
\left\{\begin{array}{l}
w^{\prime}(t)=w^{2}(t)+\frac{1}{\left(1-t^{2}\right)^{2}}, \\
w(0)=0,
\end{array}\right.
$$

whose solution is $w(t)=t\left(1-t^{2}\right)^{-1}$. Combining (16) with (17) we see that

$$
(\varphi-w)^{\prime} \leq(\varphi-w)(\varphi+w),
$$

which implies that, for $0 \leq t<1$,

$$
\left[e^{-\int_{0}^{t}(\varphi+w) d s}(\varphi-w)\right]^{\prime}=e^{-\int_{0}^{t}(\varphi+w) d s}\left[(\varphi-w)^{\prime}-(\varphi-w)(\varphi+w)\right] \leq 0 .
$$

Hence, as $\varphi(0)=w(0)$, we can get $e^{-\int(\varphi+w) d t}(\varphi-w) \leq 0$ and so, $|y|=\varphi \leq w$. This ends the proof for $\mu=1$. 
Now, we consider the case $0<\mu<1$ and, as above, let $y(t)=\partial_{z} \log \lambda(t)$ and $\varphi(t)=|y(t)|$. Proceeding as before, one finds that $y$ satisfies (15) and therefore

$$
\varphi^{\prime} \leq \varphi^{2}+\frac{\mu}{\left(1-t^{2}\right)^{2}}
$$

The difference with the proof of the first case is that now we compare with the problem

$$
\left\{\begin{array}{l}
w^{\prime}(t)=w^{2}(t)+\frac{\mu}{\left(1-t^{2}\right)^{2}}, \\
w(0)=0
\end{array}\right.
$$

whose solution is

$$
w(t)=\frac{t}{1-t^{2}}-\frac{2 \alpha^{2}}{1-t^{2}} A_{\mu}(t)
$$

where $\alpha=\sqrt{1-\mu}$ and $A_{\mu}$ is given by

$$
A_{\mu}(z)=\frac{1}{\alpha} \frac{(1+z)^{\alpha}-(1-z)^{\alpha}}{(1+z)^{\alpha}+(1-z)^{\alpha}} .
$$

From (18) and (19), it may be concluded that $|y| \leq \varphi \leq w$.

On the other hand, a straightforward calculation shows that $A_{\mu}$ is convex in $[0,1]$, so $\psi(t)=\frac{A_{\mu}(t)}{t}$ is increasing here. Thus,

$$
1-\alpha^{2} \frac{A_{\mu}(t)}{t} \leq 1-\alpha^{2} A_{\mu}^{\prime}(0)=1-\alpha^{2}=\mu .
$$

From this and the definition of $w$ we conclude the proof of the lemma.

Corollary 1. Let $0<\mu \leq 1$ and $f \in N H^{\mu}$. Then $M=2+\mu$ satisfies

$$
\left|\frac{\partial \log \lambda}{\partial z}(z)\right| \leq \frac{M}{1-|z|^{2}}
$$

for all $z \in \mathbf{D}$.

Proof. We first show the result when $\tilde{f}(\mathbf{D})$ is bounded. It is known (see [7]) that if $f \in N H$ and $\tilde{f}(\mathbf{D})$ is bounded, then

$$
u_{f}(z)=\frac{1}{\sqrt{\left(1-|z|^{2}\right) \lambda(z)}}
$$

has a critical point. Let $z_{0}$ be one of them and we define $\varphi(w)=\frac{z_{0}-w}{1-z_{0} w}$ and $g=f \circ \varphi$. Observe that $g \in N H^{\mu}$. Moreover, since $\varphi \in \operatorname{Aut}(\mathbf{D})$, we obtain from (9) that $u_{g}=u_{f} \circ \varphi$. Hence, $u_{g}$ has a critical point at zero and consequently so does $\lambda_{g}$, because of

$$
\frac{\partial_{w} u_{g}}{u_{g}}(w)=\frac{1}{2}\left\{\frac{\bar{w}}{1-|w|^{2}}-\partial_{w} \log \lambda_{g}(w)\right\} .
$$

Thus $g \in N H_{0}^{\mu}$. Lemma 1 now leads to

$$
\left|\frac{\partial \log \lambda_{g}}{\partial w}(w)\right| \leq \frac{\mu|w|}{1-|w|^{2}}, \quad w \in \mathbf{D} .
$$


On the other hand, it is easy to see that $\varphi=\varphi^{-1}$. So, $f=g \circ \varphi$ and consequently, $\lambda_{f}(z)=\lambda_{g}(\varphi(z))\left|\varphi^{\prime}(z)\right|$. Thus,

$$
\partial_{z} \log \lambda_{f}(z)=\left(\partial_{w} \log \lambda_{g}(\varphi(z))\right) \varphi^{\prime}(z)+\frac{1}{2} \frac{\varphi^{\prime \prime}}{\varphi^{\prime}}(z) .
$$

Hence, from definition of $\varphi$ and (20), we conclude that

$$
\left|\partial_{z} \log \lambda_{f}(z)\right| \leq \frac{\mu|\varphi(z)|}{1-|\varphi(z)|^{2}}\left|\varphi^{\prime}(z)\right|+\left|\frac{\overline{z_{0}}}{1-\overline{z_{0} z}}\right| \leq \frac{2+\mu}{1-|z|^{2}} .
$$

The general case follows by applying the above argument to $f_{r}(z)=\frac{1}{r} f(r z), z \in \mathbf{D}$, and letting $r \rightarrow 1^{-}$.

Corollary 2. If $f \in N H_{0}$, then for all $\xi \in \mathbf{T}$ and $0 \leq r<1$,

$$
\frac{1}{2} \lambda(r \xi) \leq \lambda(\rho \xi) \leq 2 \lambda(r \xi)
$$

$r \leq \rho \leq \frac{1+r}{2}$

Proof. Given $\xi \in \mathbf{T}$ and $0<r<\rho<1$,

$$
\log \frac{\lambda(\rho \xi)}{\lambda(r \xi)}=\int_{r}^{\rho} \frac{\partial}{\partial s} \log \lambda(s \xi) d s=\int_{r}^{\rho} 2 \operatorname{Re}\left\{\partial_{z} \log \lambda(s \xi) \xi\right\} d s .
$$

Now, by Lemma 1 we have

$$
\left|\log \frac{\lambda(\rho \xi)}{\lambda(r \xi)}\right| \leq \int_{r}^{\rho} \frac{2 s}{1-s^{2}} d s \leq \log 2
$$

if $r \leq \rho \leq \frac{1+r}{2}$, and the corollary follows.

Remark 2. By applying the same reasoning, it can be proved that

$$
\frac{1}{2^{\mu}} \lambda(r \xi) \leq \lambda(\rho \xi) \leq 2^{\mu} \lambda(r \xi)
$$

holds for all $\xi \in \mathbf{T}$ and $r \leq \rho \leq \frac{1+r}{2}$, if $f \in N H_{0}^{\mu}, 0<\mu \leq 1$. Also, under the same hypothesis on $f$, one can obtain

$$
\frac{1}{M} \lambda(r \xi) \leq \lambda(\rho \xi) \leq M \lambda(r \xi)
$$

if $0<r \leq \rho<1$ satisfy $1-r^{2} \leq M\left(1-\rho^{2}\right)$, with $M$ absolute constant.

A similar argument yields the following corollary.

Corollary 3. Let $f \in N H_{0}$ and $k>0$. If $z=r e^{i \theta}$ and $\zeta=r e^{i \nu}$ with $0<r<1$ and $|\theta-\nu| \leq k(1-r)$, then

$$
e^{-2 k} \lambda(\zeta) \leq \lambda(z) \leq e^{2 k} \lambda(\zeta)
$$

In what follows, given $f$ harmonic, we denote by $\Sigma$ the minimal surface $\tilde{f}(\mathbf{D})$ and by $d_{\Sigma}$ the metric on the surface. Moreover, $d_{f}(z)$ will denote the extended real number $d_{f}(z):=\sup \left\{r \geq 0: B_{\Sigma}(\tilde{f}(z), r) \subset \Sigma\right\}=d(\tilde{f}(z), \partial \Sigma)$. With this notation we then have the following result (see [6]). If $f$ is a harmonic function satisfying (8) with $p(t)=\left(1-t^{2}\right)^{-2}$, then

$$
d_{f}\left(z_{0}\right) \geq \frac{\left(1-\left|z_{0}\right|^{2}\right) \lambda\left(z_{0}\right)}{\frac{1-\left|z_{0}\right|}{G(1)}+\left|\left(1-\left|z_{0}\right|^{2}\right)\left(\partial_{z} \log \lambda\right)\left(z_{0}\right)-\overline{z_{0}}\right|}, \quad z_{0} \in \mathbf{D} .
$$


Here,

$$
G(r)=\frac{1}{\sqrt{2}} \frac{(1+r)^{\sqrt{2}}-(1-r)^{\sqrt{2}}}{(1+r)^{\sqrt{2}}+(1-r)^{\sqrt{2}}} .
$$

It follows from this and Corollary 1 that $C=4+\sqrt{2}$ satisfies

$$
\left(1-\left|z_{0}\right|^{2}\right) \lambda\left(z_{0}\right) \leq C d_{f}\left(z_{0}\right), \quad z_{0} \in \mathbf{D},
$$

for all $f \in N H$. Inequality (22) can be viewed as an analogue in the class $N H$ of the first inequality of

$$
\frac{1}{4}\left(1-|z|^{2}\right)\left|f^{\prime}(z)\right| \leq d_{f}(z) \leq\left(1-|z|^{2}\right)\left|f^{\prime}(z)\right|, \quad z \in \mathbf{D},
$$

which holds for $f$ analytic and univalent in the unit disk. It still is not proven an analogue of the second inequality, at least in $N H$. In this direction, Theorem 6 gives solution to the problem in $N H^{\mu}, 0<\mu<1$. In the proof of the theorem we will use some estimates whose demonstration follows the same reasoning as in the proof of Lemma 1, so we omit details.

Lemma 2. Let $g$ be a real smooth function on $(-1,1)$ such that $g^{\prime}(t)>0$ for all $t$ and $g^{\prime \prime}(0)=0$. Suppose that the Schwarzian derivative of $g$ satisfies

$$
S g(t) \leq \frac{2 \mu}{\left(1-t^{2}\right)^{2}}, \quad 0 \leq \mu<1
$$

on $(-1,1)$. Then there is $M>0$ (depending only on $\mu$ ) such that

$$
\int_{r}^{1} g^{\prime}(t) d t \leq M\left(1-r^{2}\right) g^{\prime}(r), \quad 0 \leq r<1
$$

Proof. The condition on $S g$ implies that if $y=\frac{1}{2} \frac{g^{\prime \prime}}{g^{\prime}}$, then

$$
y^{\prime}(t) \leq y^{2}(t)+\frac{2 \mu}{\left(1-t^{2}\right)^{2}}
$$

whence

$$
\frac{g^{\prime \prime}}{g^{\prime}}(t) \leq 2 \mu \frac{t}{1-t^{2}}, \quad 0 \leq t<1 .
$$

As in the proof of Corollary 2, one sees that $g^{\prime}(\rho) \leq 2^{\mu} g^{\prime}(r), 0 \leq r \leq \rho \leq(1+r) / 2$. Hence, considering the sequence $a_{0}=r$ and $a_{i}=\frac{1+a_{i-1}}{2}$, we obtain that

$$
\int_{r}^{1} g^{\prime}(t) d t=\sum_{i=1}^{\infty} \int_{a_{i-1}}^{a_{i}} g^{\prime}(t) d t \leq \sum_{i=1}^{\infty} 2^{\mu} g^{\prime}\left(a_{i-1}\right)\left(a_{i}-a_{i-1}\right) \leq(1-r) g^{\prime}(r) \sum_{i=1}^{\infty}\left(2^{\mu-1}\right)^{i} .
$$

It follows (23) with $M=\Sigma_{i=1}^{\infty}\left(2^{\mu-1}\right)^{i}$.

The same argument applied to $h(t)=-g(-t),-1<t<1$, shows that

$$
\int_{-1}^{r} g^{\prime}(t) d t \leq M\left(1-r^{2}\right) g^{\prime}(r), \quad-1<r \leq 0 .
$$

As above, $M=\sum_{i=1}^{\infty}\left(2^{\mu-1}\right)^{i}$.

Theorem 6. Let $f \in N H^{\mu}, 0<\mu<1$. There is $M>0$ (depending only on $\mu$ ) such that for all $z_{0} \in \mathbf{D}$,

$$
d_{f}\left(z_{0}\right) \leq M\left(1-\left|z_{0}\right|^{2}\right) \lambda\left(z_{0}\right) .
$$


Proof. On the one hand, since $0<\mu<1$, the strict inequality in (8) is satisfied with $p(t)=\left(1-t^{2}\right)^{-2}$. So $\tilde{f}$ is univalent in $\overline{\mathbf{D}}$ (note that $\tilde{f}(\mathbf{D}) \subset \mathbf{R}^{3}$ ). Thus, there is at most one point $\zeta$ in the boundary $\mathbf{T}$ of $\mathbf{D}$ such that $\tilde{f}(\zeta)=\infty$. Therefore there is at least a diameter $\gamma(t)=t \xi,-1<t<1$ and $\xi \in \mathbf{T}-\{ \pm \zeta\}$ such that the curve $\varphi=\tilde{f} \circ \gamma$ has finite length. By rotation, if necessary, we can assume that $\xi=1$. Thus, $\int_{-1}^{1}\left|\varphi^{\prime}(t)\right| d t<\infty$.

On the other hand, it is known that ([4], Lemma 3.1),

$$
\left.S_{1} \varphi \leq \operatorname{Re}\{\mathcal{S} f(t)\}+\lambda^{2}(t) \mid K(t)\right) \mid, \quad-1<t<1 .
$$

From this and (13) it follows that

$$
\left.\left(\frac{v^{\prime}}{v}\right)^{\prime}-\frac{1}{2}\left(\frac{v^{\prime}}{v}\right)^{2} \leq|\mathcal{S} f(t)|+\lambda^{2}(t) \mid K(t)\right) \mid,
$$

where $v(t)=\lambda(t)=\left|\varphi^{\prime}(t)\right|, t \in(-1,1)$. Thus, by definition of $N H^{\mu}, h(t)=$ $\int_{0}^{t}\left|\varphi^{\prime}(s)\right| d s$ satisfies

$$
S h(t)=\left(\frac{v^{\prime}}{v}\right)^{\prime}-\frac{1}{2}\left(\frac{v^{\prime}}{v}\right)^{2} \leq \frac{2 \mu}{\left(1-t^{2}\right)^{2}}
$$

for all $t \in(-1,1)$. Now we consider the function

$$
x(s)=\frac{e^{2 s}-1}{e^{2 s}+1}, \quad-\infty<s<\infty,
$$

which is bijective and increasing from $\mathbf{R}$ onto $(-1,1)$ with inverse $s(x)=\frac{1}{2} \log \frac{1+x}{1-x}$. We prove that $y=u \circ x$ is convex, where

$$
u(t):=u_{h}(t)=\frac{1}{\sqrt{\left(1-t^{2}\right) h^{\prime}(t)}}, \quad-1<t<1 .
$$

In first place, note that $y=(w \circ x) / \sqrt{x^{\prime}}$. Here, $w=1 / \sqrt{h^{\prime}}$, which is a solution of

$$
w^{\prime \prime}+\frac{1}{2}(S h) w=0 .
$$

By deriving $\log y$ and noting that $x^{\prime}=1-x^{2}$, one obtains

$$
\frac{y^{\prime \prime}}{y}-\left(\frac{y^{\prime}}{y}\right)^{2}=\frac{\left(w^{\prime} \circ x\right) x^{\prime \prime}}{w \circ x}+\left(x^{\prime}\right)^{2} \frac{w^{\prime \prime} \circ x}{w \circ x}-\frac{\left(w^{\prime} \circ x\right)^{2}\left(x^{\prime}\right)^{2}}{(w \circ x)^{2}}+x^{\prime} .
$$

Hence, by (28) and $x^{\prime \prime}=-2 x x^{\prime}$, we see that $y$ satisfies

$$
y^{\prime \prime}(s)=\left(1-\left(1-x^{2}(s)\right)^{2} \frac{1}{2} S h(x(s))\right) y .
$$

Finally, by (27) the expression in parenthesis is non negative, so $y$ is convex.

Now we will show that $u$ has an absolute minimum. In fact, since the integral

$$
\int_{0}^{1}\left|\varphi^{\prime}(t)\right| d t=\int_{0}^{1} h^{\prime}(t) d t=\int_{0}^{\infty} h^{\prime}(x(s)) x^{\prime}(s) d s=\int_{0}^{\infty} \frac{d s}{y^{2}(s)}
$$

is finite, there is an increasing and non bounded sequence $\left(s_{n}\right)$ such that $y\left(s_{n}\right) \rightarrow \infty$ when $n \rightarrow \infty$. The convexity of $y$ implies that $y(s) \rightarrow \infty$ when $s \rightarrow \infty$. Hence, since $y(s)=u(x(s))$ and $x(s) \rightarrow 1$ if and only if $s \rightarrow \infty$, we conclude that $u(t) \rightarrow \infty$ when $t \rightarrow 1$. Similarly, $u(t) \rightarrow \infty$ when $t \rightarrow-1$. 
Now we can claim that there is $t_{0} \in(-1,1)$ such that $u^{\prime}\left(t_{0}\right)=0$ and we proceed as in the proof of Corollary 1. Without loss of generality we suppose $t_{0} \geq 0$ and we consider the function $T(s)=\frac{t_{0}-s}{1-t_{0} s}$, which is bijective from $(-1,1)$ onto $(-1,1)$, decreasing, and satisfies $\left(1-|s|^{2}\right)\left|T^{\prime}(s)\right|=1-|T(s)|^{2}$. It follows that, if $g=-h \circ T$, then $u_{g}(s)=u_{h}(T(s))$ and therefore $u_{g}^{\prime}(0)=u_{h}^{\prime}(T(0)) T^{\prime}(0)=0$. Thus, the equality

$$
\frac{u_{g}^{\prime}(s)}{u_{g}(s)}=\frac{s}{1-s^{2}}-\frac{1}{2} \frac{g^{\prime \prime}(s)}{g^{\prime}(s)}
$$

implies $g^{\prime \prime}(0)=0$. Observe that, from the definition of $g$ and (27),

$$
S g(s)=(S h \circ T(s))\left(T^{\prime}(s)\right)^{2} \leq \frac{2 \mu}{\left(1-|s|^{2}\right)^{2}},
$$

$-1<s<1$. By Lemma 2 we deduce that $g$ satisfies (23) and (25).

Let us first prove (26) when $z_{0}$ is a real number $r$.

Step 1. Suppose that $r \leq t_{0}$. By definition of $g$, one easily has that

$$
d_{f}(r) \leq \int_{-1}^{r} h^{\prime}(t) d t \leq \int_{1}^{T^{-1}(r)} h^{\prime}(T(s)) T^{\prime}(s) d s=\int_{T^{-1}(r)}^{1} g^{\prime}(s) d s .
$$

But $T^{-1}(r) \geq T^{-1}\left(t_{0}\right)=0$ since $T$ is decreasing. So, by (23),

$$
d_{f}(r) \leq M\left(1-\left|T^{-1}(r)\right|^{2}\right) g^{\prime}\left(T^{-1}(r)\right) .
$$

Hence a straightforward calculation shows that

$$
d_{f}(r) \leq M\left(1-r^{2}\right) h^{\prime}(r)=M\left(1-r^{2}\right) \lambda(r)
$$

and follows Step 1.

Step 2. Suppose now $r \geq t_{0}$ and let $S(\zeta)=\frac{r-\zeta}{1-r \zeta}$ and $F=f \circ S$. Since $S \in \operatorname{Aut}(\mathbf{D})$, $F \in N H^{\mu}$. Moreover, $\lambda_{F}=\left(\lambda_{f} \circ S\right)\left|S^{\prime}\right|$ and, as $r$ is real, $S$ fixes the diameter $(-1,1)$. Thus, the curve $\psi(t)=\tilde{F}(t),-1<t<1$ has finite length. If $h_{1}$ denotes the arc length function of $\psi$, then

$$
h_{1}^{\prime}(t)=\left|\psi^{\prime}(t)\right|=\lambda_{F}(t)=\lambda_{f}(S(t))\left|S^{\prime}(t)\right|=h^{\prime}(S(t))\left|S^{\prime}(t)\right|,
$$

whence we obtain

$$
u_{h_{1}}(t)=\frac{1}{\sqrt{\left(1-t^{2}\right) h_{1}^{\prime}(t)}}=\frac{1}{\sqrt{\left(1-t^{2}\right) h^{\prime}(S(t))\left|S^{\prime}(t)\right|}}=u_{h}(S(t))
$$

and consequently, $S^{-1}\left(t_{0}\right)$ is a critical point of $u_{h_{1}}$. Now, as $S^{-1}\left(t_{0}\right)=\frac{r-t_{0}}{1-r t_{0}} \geq 0$ because $r \geq t_{0}$, it follows from Step 1 that $d_{F}(s) \leq M\left(1-s^{2}\right) h_{1}^{\prime}(s)$ for all $s \leq S^{-1}\left(t_{0}\right)$. If in particular $s=0$, one then has that

$$
d_{f}(r)=d_{F}(0) \leq M h_{1}^{\prime}(0)=M\left(1-r^{2}\right) h^{\prime}(r)
$$

and we conclude Step 2. Thus, we have proven $(26)$ if $z_{0} \in(-1,1)$.

Finally, as $\tilde{f}$ is injective from $\mathbf{D}$ into $\mathbf{R}^{3} \cup\{\infty\}$, there is at most one $\zeta \in \mathbf{T}$ such that $\tilde{f}(\zeta)=\infty$. So, given $z_{0} \in \mathbf{D}$, there is $\xi \in \mathbf{T}$ such that if $\gamma$ is the hyperbolic segment determined by $\xi$ and $z_{0}$, then the curve $\varphi=\tilde{f} \circ \gamma$ has finite length. Fix an automorphism $T$ of $\mathbf{D}$ that maps $(-1,1)$ onto $\gamma$ with the conditions $T(0)=z_{0}$ and $\left|T^{\prime}(0)\right|=1-\left|z_{0}\right|^{2}$ and we define the harmonic function $F=f \circ T$. We know that 
$F \in N H^{\mu}$ and the curve $\psi(t)=\tilde{F}(t),-1<t<1$ has finite length. From the above proven it follows that

$$
d_{F}(r) \leq M\left(1-r^{2}\right) \lambda_{F}(r), \quad-1<r \leq 1 .
$$

But $\lambda_{F}=(\lambda \circ T)\left|T^{\prime}\right|$, then for $r=0$ one obtains that

$$
d_{f}\left(z_{0}\right)=d_{F}(0) \leq M \lambda_{F}(0)=M \lambda_{f}(T(0))\left|T^{\prime}(0)\right|=M\left(1-\left|z_{0}\right|^{2}\right) \lambda_{f}\left(z_{0}\right),
$$

which completes the proof.

\section{Proof of the main results}

In this section we will prove the main theorems of the paper. We start with some results concerning to the classes of John surfaces defined before.

Proof of Theorem 1. a.) Given $q \in \Sigma, q \neq p$, there are $\xi \in \mathbf{T}$ and $\rho \in(0,1)$ such that $q=\tilde{f}(\rho \xi)$. The curve $\gamma_{\xi}(t)=\tilde{f}(t \xi), 0 \leq t \leq \rho$ has endpoints at $p$ and $q$ and moreover, if $y$ is a point of the curve, then $y=\tilde{f}(r \xi)$ for some $r \in(0, \rho)$. Proceeding as in the proof of (23), one sees that

$$
\ell\left(\gamma_{\xi}(y, q)\right) \leq M\left(1-r^{2}\right) \lambda(r \xi)
$$

where $M=\sum_{i=1}^{\infty}\left(2^{\mu-1}\right)^{i}>1$. It follows from this and (22), that

$$
\ell\left(\gamma_{\xi}(y, q)\right) \leq b d_{f}(r \xi)=b d_{\Sigma}(y, \partial \Sigma),
$$

with $b=M C>1$ and we conclude a.).

b.) Fix $\xi \in \mathbf{T}$. From above we know that $\varphi(r)=\int_{r}^{1} \lambda(t \xi) d t$ satisfies

$$
\varphi(r) \leq M\left(1-r^{2}\right) \lambda(r \xi), \quad M>1 .
$$

This inequality and $\varphi^{\prime}(r)=-\lambda(r \xi)$ imply that, for $0<r<\rho<1$,

$$
\log \frac{\varphi(\rho)}{\varphi(r)}=\int_{r}^{\rho} \frac{\varphi^{\prime}(t)}{\varphi(t)} d t \leq \frac{1}{2 M} \log \frac{1-\rho}{1-r} .
$$

Thus,

$$
\varphi(\rho) \leq \varphi(r)\left(\frac{1-\rho}{1-r}\right)^{1 / 2 M} .
$$

On the other hand, by definition of $\varphi$ and by Corollary 2 ,

$$
\varphi(\rho) \geq \int_{\rho}^{(1+\rho) / 2} \lambda(t \xi) d t \geq \frac{1}{4}(1-\rho) \lambda(\rho \xi),
$$

whence $\varphi(\rho) \geq \frac{1}{8}\left(1-\rho^{2}\right) \lambda(\rho \xi)$. Hence, by (29) and (30),

$$
\frac{1}{8}\left(1-\rho^{2}\right) \lambda(\rho \xi) \leq M\left(1-r^{2}\right) \lambda(r \xi)\left(\frac{1-\rho}{1-r}\right)^{1 / 2 M}
$$

and therefore

$$
\frac{\left(1-\rho^{2}\right) \lambda(\rho \xi)}{\left(1-r^{2}\right) \lambda(r \xi)} \leq K\left(\frac{1-\rho}{1-r}\right)^{\alpha},
$$

where $\alpha=\frac{1}{2 M} \in(0,1)$ and $K=8 M$. 
c.) Let $\zeta=\rho e^{i \theta} \in B(z)$. From Corollary 3 and $b$.) it follows that

$$
\frac{\left(1-|\zeta|^{2}\right) \lambda(\zeta)}{\left(1-|z|^{2}\right) \lambda(z)} \leq e^{\pi} \frac{\left(1-\rho^{2}\right) \lambda\left(\rho e^{i t}\right)}{\left(1-r^{2}\right) \lambda\left(r e^{i t}\right)} \leq e^{\pi} K\left(\frac{1-\rho}{1-r}\right)^{\alpha}
$$

which implies (11) with $K_{1}=e^{\pi} K$.

Corollary 4. Let $0<\mu<1$ and $f \in N H^{\mu}$ be bounded. Then $\tilde{f}(\mathbf{D})$ is a John surface.

Proof. With the notation of Corollary $1, g=f \circ \varphi \in N H_{0}^{\mu}$. From above theorem, we get immediately that $\tilde{f}(\mathbf{D})=\tilde{g}(\mathbf{D})$ is a John surface.

In general, if $f \in N H, \tilde{f}(\mathbf{D})$ need not be a John surface. Now we will prove that it is true when $f$ satisfies (10).

Theorem 7. Let $f \in N H$ and we suppose that there are $\alpha \in(0,1), r_{0} \in[0,1)$, and $K>0$ satisfying (10) for $0 \leq r_{0} \leq r<\rho<1$. That is,

$$
\frac{\left(1-\rho^{2}\right) \lambda(\rho \xi)}{\left(1-r^{2}\right) \lambda(r \xi)} \leq K\left(\frac{1-\rho}{1-r}\right)^{\alpha},
$$

for all $\xi \in \mathbf{T}$ and $0 \leq r_{0} \leq r<\rho<1$. Then $\Sigma=\tilde{f}(\mathbf{D})$ is a John surface with center $\tilde{f}(0)=p$ and the curves $\gamma_{\xi}(t)=\tilde{f}(t \xi), 0 \leq t \leq 1$ are John curves.

Proof. First we note that by compactness of $\left\{z:|z| \leq r_{0}\right\}$ and continuity of $\lambda$, there is a constant $\bar{K}>0$, which also will be denoted by $K$, satisfying (10) for $0 \leq r<\rho<1$.

Next, we prove that $\Sigma$ is bounded. The condition (10) with $r=0$ implies that $\lambda(\rho \xi) \leq K_{1}(1-\rho)^{\alpha-1}, \xi \in \mathbf{T}$ and $0<\rho<1$. Hence, given any point $\tilde{f}(\rho \xi)$ in $\Sigma$,

$$
|\tilde{f}(\rho \xi)-\tilde{f}(0)| \leq \int_{0}^{\rho} \lambda(t \xi) d t \leq \frac{K_{1}}{\alpha}
$$

and therefore $\Sigma$ is bounded.

On the other hand, by Corollary 1 there is a constant $M$, which is independent of $f$, such that

$$
\left|\frac{\partial \log \lambda}{\partial z}(z)\right| \leq \frac{M}{1-|z|^{2}}, \quad z \in \mathbf{D}
$$

Hence, by $(21)$ we deduce that $C=1+\sqrt{2}+M$ satisfies

$$
\left(1-|z|^{2}\right) \lambda(z) \leq C d_{f}(z), \quad z \in \mathbf{D} .
$$

Finally, fix $q \in \Sigma, q \neq p$, and let $\xi \in \mathbf{T}$ and $\rho \in(0,1)$ such that $q=\tilde{f}(\rho \xi)$. The curve $\gamma_{\xi}(t)=\tilde{f}(t \xi), 0 \leq t \leq \rho$ has endpoints at $p$ and $q$ and, if $y$ is a point of the curve, $y=\tilde{f}(r \xi)$ for some $r \in(0, \rho)$. By (10) one obtains that

$$
\ell\left(\gamma_{\xi}(y, q)\right)=\int_{r}^{\rho} \lambda(t \xi) d t \leq K(1-r)^{1-\alpha} \lambda(r \xi) \int_{r}^{\rho} \frac{d t}{(1-t)^{1-\alpha}}
$$

whence

$$
\ell\left(\gamma_{\xi}(y, q)\right) \leq \frac{K}{\alpha}\left(1-r^{2}\right) \lambda(r \xi) \leq b d_{f}(r \xi)
$$

with $b=K C / \alpha$. 
As we have mentioned in the introduction, in [10] it is proven that if $f \in N$ satisfies (3), then $f(\mathbf{D})$ is a John domain. The following theorem generalizes this result to harmonic mappings.

Theorem 8. Let $f \in N H$. If

$$
\limsup _{|z| \rightarrow 1}\left(1-|z|^{2}\right)\left|\partial_{z} \log \lambda(z)\right|<1,
$$

then $\Sigma=\tilde{f}(\mathbf{D})$ is a John surface with center $p=\tilde{f}(0)$. Furthermore, the curves $\gamma_{\xi}(t)=\tilde{f}(t \xi)$ are John curves for every $\xi \in \mathbf{T}$.

Proof. By (32) there is a constant $M$ for which (31) holds. Thus, from the proof of Theorem 7, it is sufficient to show that there is $r_{0}$ satisfying (10) for $0<r_{0} \leq r<$ $\rho<1$. In fact, by (32), there are $0<\beta<1$ and $\beta \leq r_{0}<1$ such that

$$
\left(1-|z|^{2}\right)\left|\partial_{z} \log \lambda(z)\right| \leq \beta<1, \quad r_{0} \leq|z|<1 .
$$

Thus, for $r_{0} \leq r<\rho<1$ and $\xi \in \mathbf{T}$, we have

$$
\begin{aligned}
\log \frac{\left(1-\rho^{2}\right)^{2} \lambda(\rho \xi)}{\left(1-r^{2}\right) \lambda(r \xi)} & =\int_{r}^{\rho} \frac{\partial}{\partial t} \log \left(1-t^{2}\right) \lambda(t \xi) d t \\
& =2 \int_{r}^{\rho} \operatorname{Re}\left\{\xi \partial_{z} \log \lambda(t \xi)-\frac{t}{1-t^{2}}\right\} d t<\int_{r}^{\rho} \frac{-2 \alpha}{1-t^{2}} d t
\end{aligned}
$$

where $\alpha=r_{0}-\beta$. Hence,

$$
\log \frac{\left(1-\rho^{2}\right)^{2} \lambda(\rho \xi)}{\left(1-r^{2}\right) \lambda(r \xi)} \leq \log \left(\frac{1-\rho}{1-r}\right)^{\alpha}\left(\frac{1+r}{1+\rho}\right)^{\alpha}
$$

whence (10) holds for $0<r_{0} \leq r \leq \rho<1$. This completes the proof.

Proof of Theorem 2. The demonstration is an application of the results proved for the class $N H^{\mu}, 0<\mu<1$ and follows the argument of the proof of Theorem 4 in [15]. We will use the same notation as in that proof.

The condition on $c$ implies that there are $\delta>0$ and $0<r_{1}<1$ such that

$$
|\mathcal{S} f(z)|+\lambda^{2}(z)|K(z)| \leq \frac{2-5 \delta}{\left(1-|z|^{2}\right)^{2}}
$$

holds in the annulus $\left\{r_{1}<|z|<1\right\}$. Next, for $\alpha>0$ to be chosen later, we consider the function

$$
\varphi(\xi):=\varphi_{\alpha}(\xi)=e^{-i \pi \delta / 2}\left(\frac{1+\xi}{1-\xi}\right)^{1-\delta}-i \alpha
$$

whose Schwarzian derivative is given by

$$
S_{\varphi}(\xi)=\frac{2 \delta(2-\delta)}{\left(1-\xi^{2}\right)^{2}}, \quad \xi \in \mathbf{D} .
$$

Note that $\varphi$ maps the unit disk conformally onto a domain $\Omega \subset\{z: \operatorname{Re} z>0\}$. $\Omega$ is an angle with vertex $-i \alpha$, measure $\pi(1-\delta)$, and has $[-i \alpha,-i \infty]$ as one boundary line. Now it is easily seen that the function

$$
\psi(\xi)=e^{i \theta} \frac{\varphi(\xi)-1}{\varphi(\xi)+1}=T \circ \varphi, \quad 0 \leq \theta \leq 2 \pi,
$$

where $T(z)=e^{i \theta \frac{z-1}{z+1}}$, maps the unit disk onto a subdomain $H$ of $\mathbf{D}$, bounded by an arc of $\mathbf{T}$ (the image of the ray $[-i \alpha,-i \infty]$ under $\psi$ ) and a circular segment $S$ contained 
in the interior of the unit disk. $S$ intersects to $\mathbf{T}$ at $e^{i \theta}$ and $e^{i \theta}(\alpha-i) /(\alpha+i)$. See Figure 3.1.
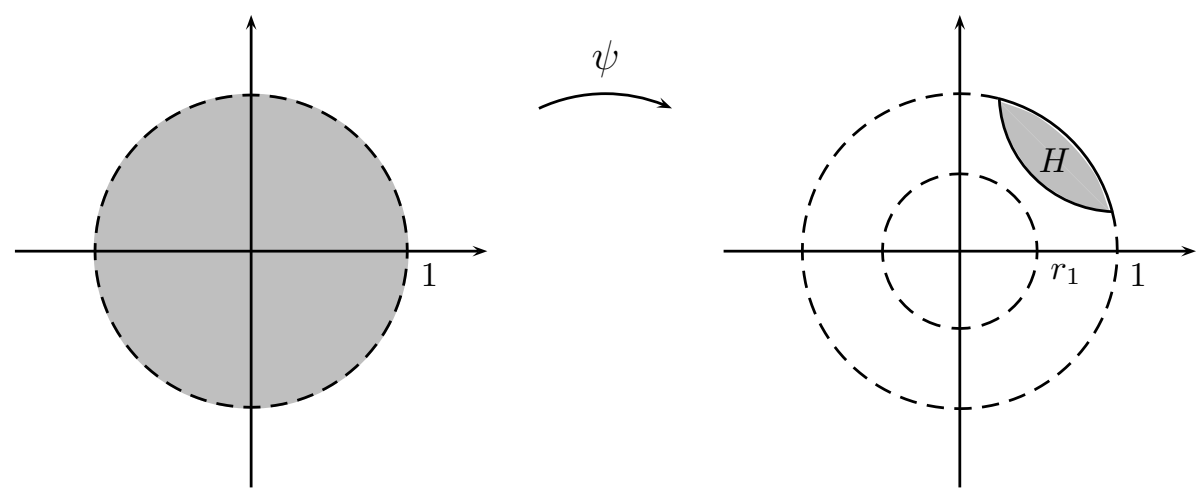

Figure 3.1.

Now, since the argument of $(\alpha-i) /(\alpha+i)$ is negative, $\partial H \cap \mathbf{T}$ has the form $\left\{e^{i t}: \theta-\beta \leq t \leq \theta\right\}$ with $\beta$ depending only on $\alpha$. As the angle in the intersection $(\pi(1-\delta))$ and one of the points of intersection are independent of $\alpha$, we can choose $\alpha$ in such a way that $H \subset\left\{r_{1}<|z|<1\right\}$. Proceeding in this way one can construct finitely many congruent domains $H:=H(\theta)$ which cover an annulus of the form $\left\{r_{2}<|z|<1\right\}$, where $0<r_{1}<r_{2}<1$. Let $H_{1}, \ldots, H_{n}$ be such domains.

On the other hand, if $H$ is one of the domains $H_{1}, \ldots, H_{n}$ and $\psi$ is the corresponding function, we obtain from (34) and $\psi=T \circ \varphi$ that

$$
S_{\psi}(\xi)=S_{\varphi}(\xi)=\frac{2 \delta(2-\delta)}{\left(1-\xi^{2}\right)^{2}}, \quad \xi \in \mathbf{D}
$$

Thus, as $H=\psi(\mathbf{D}) \subset\left\{r_{1}<|z|<1\right\}$, it follows from (6), (9), (33), and (35) that the harmonic function $h=f \circ \psi$ satisfies

$$
\begin{aligned}
|\mathcal{S} h(\xi)|+\lambda_{h}^{2}(\xi)\left|K_{h}(\xi)\right| & \leq|\mathcal{S} f(\psi(\xi))|\left|\psi^{\prime}(\xi)\right|^{2}+\left|S_{\psi}(\xi)\right|+\lambda_{f}^{2}(\psi(\xi))\left|\psi^{\prime}(\xi)\right|^{2}\left|K_{f}(\psi(\xi))\right| \\
& \leq \frac{2-5 \delta}{\left(1-|\psi(\xi)|^{2}\right)^{2}}\left|\psi^{\prime}(\xi)\right|^{2}+\frac{2 \delta(2-\delta)}{\left(1-|\xi|^{2}\right)^{2}} .
\end{aligned}
$$

Hence by Schwarz-Pick's Lemma,

$$
|\mathcal{S h}(\xi)|+\lambda_{h}^{2}(\xi)\left|K_{h}(\xi)\right| \leq \frac{2-\delta}{\left(1-|\xi|^{2}\right)^{2}}
$$

and therefore $h \in N H^{\mu}$, where $\mu=(2-\delta) / 2<1$. We conclude from Corollary 4 that $\tilde{f}(H)=\tilde{h}(\mathbf{D})$ is a John surface.

Thus, we have built finitely many domains $H_{1}, \ldots, H_{n}$ covering an annulus of the form $\left\{r_{2} \leq|z|<1\right\}$ such that, for all $k=1, \ldots, n, \tilde{f}\left(H_{k}\right)$ is a $b_{k^{-}}$John surface for some $b_{k}>1$.

We now complete the proof of the theorem as follows. We denote by $p_{k}=$ $\tilde{f}\left(r_{k} \xi_{k}\right), 0<r_{k}<1$, and $\xi \in \mathbf{T}$ the center of the John surface $\tilde{f}\left(H_{k}\right)$ and let $\Sigma_{2}=\tilde{f}\left(\left\{z:|z| \leq r_{2}\right\}\right), M=\max \left\{\lambda(z):|z| \leq r_{2}\right\}$, and $\alpha_{k}(t)=\tilde{f}\left(t \xi_{k}\right), 0 \leq t \leq r_{k}$. 
Given $q \in \Sigma_{2}, q \neq p=\tilde{f}(0)$, there is $\xi \in \mathbf{T}$ and $0 \leq r \leq r_{2}$ such that $q=\tilde{f}(r \xi)$. Thus, $\gamma(t)=\tilde{f}(t \xi), 0 \leq t \leq r$ has endpoints at $p$ and $q$ and, if $y \in \gamma$,

$$
\ell(\gamma(y, q)) \leq \int_{0}^{r_{2}} \gamma(t \xi) d t \leq \frac{M}{K_{1}} d_{f}(y)
$$

where $K_{1}=\inf \left\{d_{f}(z):|z| \leq r_{2}\right\}>0$.

Next, we consider the case $q \notin \Sigma_{2}$. Then $q \in \tilde{f}\left(H_{k}\right)$ for some $k \in\{1,2 \ldots, n\}$. Let $\alpha$ be a John curve from $p_{k}$ to $q$ and $\gamma:=\gamma_{k}=\alpha_{k}+\alpha$. We have two cases. If $y \in \alpha$,

$$
\ell(\gamma(y, q))=\ell(\alpha(y, q)) \leq b_{k} d(y, \partial \Sigma) .
$$

Now we suppose that $y$ is any point in $\alpha_{k}$. The compactness of $\alpha_{1} \cup \ldots \cup \alpha_{n}$ implies that there is $K_{2}>0$ satisfying

$$
d(z, \partial \Sigma) \leq K_{2} \quad \text { for all } z \in \alpha_{1} \cup \ldots \cup \alpha_{n} .
$$

Then, if $K_{3}=\max \left\{\ell\left(\alpha_{k}\right)+b_{k} d\left(p_{k}, \partial \Sigma\right): k=1, \ldots n\right\}$,

$$
\ell\left(\gamma_{k}(y, q)\right) \leq \ell\left(\alpha_{k}\right)+\ell(\alpha) \leq I+b_{k} d\left(p_{k}, \partial \Sigma\right) \leq K_{3} .
$$

Hence,

$$
\ell\left(\gamma_{k}(y, q)\right) \leq \frac{K_{3}}{K_{2}} d_{f}(y) .
$$

It follows that $\tilde{f}(\mathbf{D})$ is a $b$-John surface with $b=\max \left\{M / K_{1}, K_{3} / K_{2}, b_{k}\right\}$.

We proceed now with the proof of Theorem 3. Before the proof, we need some preliminary lemmas.

Lemma 3. Let $a=r e^{i \theta} \in \mathbf{D}$ and $S$ be the hyperbolic segment orthogonal to diameter $\left[-e^{i \theta}, e^{i \theta}\right]$ at $a$. Let $e^{i \theta_{1}}$ and $e^{i \theta_{2}}, \theta_{1}<\theta_{2}$ be the endpoints of $S$. There is a constant $K$, which is independent of $r$, such that for all $w=r_{1} e^{i \alpha} \in S$ holds

a.) $\left|\theta_{2}-\alpha\right| \leq K\left(1-r_{1}\right)$, if $\theta \leq \alpha$;

b.) $\left|\theta_{1}-\alpha\right| \leq K\left(1-r_{1}\right)$, if $\alpha \leq \theta$.

Proof. Without loss of generality, we assume that $\alpha>\theta=0$.

Let $\beta=\angle\left(w, e^{i \theta_{2}}, e^{i \alpha}\right)$ and let $\gamma=\angle\left(r, e^{i \theta_{2}}, 1\right)$. Then $\beta \geq \gamma$. But $\gamma=\pi / 4$, since

$$
\gamma=\pi / 2-\angle\left(0, e^{i \theta_{2}}, r\right)-\angle\left(1, e^{i \theta_{2}}, c\right)
$$

where $c$ is the center of $S$, and further

$$
\angle\left(0, e^{i \theta_{2}}, r\right)+\angle\left(1, e^{i \theta_{2}}, c\right)=\frac{1}{2}(\varphi+\phi),
$$

where $\varphi$ and $\phi$ are the two other angles of right triangle $\triangle\left(0, e^{i \theta_{2}}, c\right)$.

Now, let $z$ be the projection of $e^{i \alpha}$ on the segment $\left[w, e^{i \theta_{2}}\right]$. Then, since $\beta \geq \pi / 4$, we have $\left|e^{i \theta_{2}}-e^{i \alpha}\right| \leq \sqrt{2}\left|z-e^{i \alpha}\right|$. Combining this with

$$
\left|\theta_{2}-\alpha\right| \leq \frac{\pi}{2 \sqrt{2}}\left|e^{i \theta_{2}}-e^{i \alpha}\right| \text { and }\left|z-e^{i \alpha}\right| \leq\left|w-e^{i \alpha}\right|
$$

we obtain the result with $K=\pi / 2$.

Remark 3. With the notation before, we can conclude from Lemma 3 and Corollary 3 that there is $M>0$ such that if $f \in N H_{0}$, then

a.) $\frac{1}{M} \lambda\left(r_{1} e^{i \theta_{2}}\right) \leq \lambda(w) \leq M \lambda\left(r_{1} e^{i \theta_{2}}\right)$, if $\theta \leq \alpha$;

b.) $\frac{1}{M} \lambda\left(r_{1} e^{i \theta_{1}}\right) \leq \lambda(w) \leq M \lambda\left(r_{1} e^{i \theta_{1}}\right)$, if $\theta \geq \alpha$. 
Lemma 4. With the notation of Lemma 3, if $f \in N H_{0}^{\mu}, 0<\mu<1$, then

$$
\ell(\Gamma) \leq \widetilde{M}\left(1-r^{2}\right) \lambda(a),
$$

where $\Gamma=\tilde{f}(S)$ and $\widetilde{M}$ is a constant depending only on $\mu$.

Proof. Let $S_{i} \subset S$ be the arc from $a$ to $e^{i \theta_{i}}$ and $\Gamma_{i}=\tilde{f}\left(S_{i}\right), i=1,2$. We will have proved the lemma if we show that, for $i=1,2 \ell\left(\Gamma_{i}\right) \leq \widetilde{M}_{i}\left(1-r^{2}\right) \lambda(a)$ with $\widetilde{M}_{i}$ depending only on $\mu$. We will prove only the case $i=2$, because the proof of the other one is similar. For this we consider the parametrization of $S_{2}$ given by

$$
\varphi(t)=\xi \frac{r i-t}{1+r t i}, \quad 0 \leq t \leq 1
$$

where $\xi=-i e^{i \theta}$. Hence by Remark 3 ,

$$
\ell\left(\Gamma_{2}\right)=\int_{0}^{1} \lambda(\varphi(t))\left|\varphi^{\prime}(t)\right| d t \leq M \int_{0}^{1} \lambda\left(|\varphi(t)| e^{i \theta_{2}}\right)\left|\varphi^{\prime}(t)\right| d t
$$

To obtain the lemma we consider separately the two integrals

$$
\int_{0}^{1 / 2} \lambda\left(|\varphi(t)| e^{i \theta_{2}}\right)\left|\varphi^{\prime}(t)\right| d t \text { and } \int_{1 / 2}^{1} \lambda\left(|\varphi(t)| e^{i \theta_{2}}\right)\left|\varphi^{\prime}(t)\right| d t .
$$

For the first integral, we see from

$$
1-|\varphi(t)|^{2} \geq 1-\left|\varphi\left(\frac{1}{2}\right)\right|^{2} \geq 1-\frac{r^{2}+\left(\frac{1}{2}\right)^{2}}{1+\left(\frac{r}{2}\right)^{2}}
$$

that $1-r^{2} \leq 2\left(1-|\varphi(t)|^{2}\right)$ holds for $0 \leq t \leq \frac{1}{2}$. So in view of Remark 2 , we conclude that there is $M_{2}$ such that

$$
\int_{0}^{1 / 2} \lambda\left(|\varphi(t)| e^{i \theta_{2}}\right)\left|\varphi^{\prime}(t)\right| d t \leq M_{2} \int_{0}^{1 / 2} \lambda\left(r e^{i \theta_{2}}\right)\left|\varphi^{\prime}(t)\right| d t .
$$

Finally, from Remark 3 and the inequality $\left|\varphi^{\prime}(t)\right| \leq 4\left(1-r^{2}\right)$, which holds for $0 \leq$ $t \leq \frac{1}{2}$, it follows that

$$
\int_{0}^{1 / 2} \lambda\left(|\varphi(t)| e^{i \theta_{2}}\right)\left|\varphi^{\prime}(t)\right| d t \leq M_{3}\left(1-r^{2}\right) \lambda(a) .
$$

For the second integral, defining $u=|\varphi(t)|$ one verifies that

$$
u u^{\prime}=t \frac{1+r^{2}}{1+r^{2} t^{2}}\left|\varphi^{\prime}(t)\right| \text { and } t^{2}=\frac{u^{2}-r^{2}}{1-r^{2} u^{2}},
$$

hence

$$
\left|\varphi^{\prime}(t)\right|=\frac{1}{t\left(1+r^{2}\right)} \sqrt{1+r^{2} t^{2}} \sqrt{t^{2}+r^{2}} u^{\prime} \leq 4 u^{\prime}
$$

and finally

$$
\int_{1 / 2}^{1} \lambda\left(|\varphi(t)| e^{i \theta_{2}}\right)\left|\varphi^{\prime}(t)\right| d t \leq 4 \int_{|\varphi(1 / 2)|}^{1} \lambda\left(u e^{i \theta_{2}}\right) d u \leq 4 \int_{r}^{1} \lambda\left(u e^{i \theta_{2}}\right) d u .
$$

Proceeding now as in the proof of (23) we obtain

$$
\int_{1 / 2}^{1} \lambda\left(|\varphi(t)| e^{i \theta_{2}}\right)\left|\varphi^{\prime}(t)\right| d t \leq M_{4}\left(1-r^{2}\right) \lambda\left(r e^{i \theta_{2}}\right)
$$


with $M_{4}$ depending only on $\mu$. The lemma follows by using Remark 3 and (36).

Lemma 5. Let $M, \delta$, and $C$ be positive constants. The family $\mathcal{F}$ of harmonic functions $f=h+\bar{g}$ such that $h(0)=g(0)=0, \delta \leq \lambda(0) \leq C$, and

$$
\left|\frac{\partial \log \lambda}{\partial z}(z)\right| \leq \frac{M}{1-|z|^{2}}, \quad z \in \mathbf{D},
$$

is compact. Here, as before, $\lambda(z)=\left|h^{\prime}(z)\right|+\left|g^{\prime}(z)\right| \neq 0$ for all $z \in \mathbf{D}$.

Proof. Given $z \in \mathbf{D}$, by (37) and a standard argument of integration one obtains for $f \in \mathcal{F}$ that,

$$
\delta_{1}(1-|z|)^{M} \leq \lambda(z) \leq \frac{C_{1}}{(1-|z|)^{M}} .
$$

Hence, by (37), it follows that

$$
|\nabla \lambda(z)| \leq \frac{2 C_{1} M}{(1-|z|)^{M+1}}
$$

whence $\left\{\lambda_{f}: f \in \mathcal{F}\right\}$ is a normal family. Note moreover that (38) and $\lambda=\left|h^{\prime}\right|+$ $\left|g^{\prime}\right|$ imply that $\{h: f=h+\bar{g} \in \mathcal{F}\}$ and $\{g: f=h+\bar{g} \in \mathcal{F}\}$ are normal families of analytic functions. Thus, given the sequences of functions $f_{n}=h_{n}+\overline{g_{n}} \in \mathcal{F}$ and $\lambda_{n}=\left|h_{n}^{\prime}\right|+\left|g_{n}^{\prime}\right|$, there are analytic functions $h, g$, a nonnegative function $\lambda$, and subsequences $\left(h_{n_{k}}\right),\left(g_{n_{k}}\right)$, and $\left(\lambda_{n_{k}}\right)$ such that

$$
h_{n_{k}} \rightarrow h, \quad g_{n_{k}} \rightarrow g, \quad \text { and } \quad \lambda_{n_{k}} \rightarrow \lambda
$$

locally uniformly in D. It follows that if $f=h+\bar{g}$, then $\lambda_{f}=\lambda$. Furthermore, in view of (38) we deduce that $\lambda_{f}$ does not vanish in $\mathbf{D}$. It is clear also that $\lambda_{f}$ satisfies (37) and $f_{n_{k}} \rightarrow f$ locally uniformly in $\mathbf{D}$. In consequence, $f \in \mathcal{F}$ and so $\mathcal{F}$ is compact.

Lemma 6. Let $a$ and $S$ be as in Lemma 3 and $T$ the automorphism of the unit disk that maps $(-1,1)$ onto $S$ in such a way that, $T(-1)=e^{i \theta_{2}}, T(0)=a$, and $T(1)=e^{i \theta_{1}}$. Suppose moreover that $f \in N H_{0}^{\mu}, 0<\mu<1$ and $\lambda_{1}=(\lambda \circ T)\left|T^{\prime}\right|$. If $x$ is a critical point of the function

$$
v(t)=\frac{1}{\sqrt{\left(1-t^{2}\right) \lambda_{1}(t)}}, \quad-1<t<1,
$$

and $|x|>\mu+\eta$, for some $\eta>0$, then there is $M>0$ such that

$$
\frac{1}{M} \lambda(y) \leq \lambda(a) \leq M \lambda(y)
$$

where $y=T(x)$.

Proof. By a straightforward calculation one can see that $T(z)=-i e^{i \theta} \frac{r i+z}{1-r i z}$,

$$
\frac{v^{\prime}(t)}{v(t)}=\frac{1}{2}\left\{\frac{2 t}{1-t^{2}}-\frac{\lambda_{1}^{\prime}(t)}{\lambda_{1}(t)}\right\},
$$

and

$$
\frac{\lambda_{1}^{\prime}(t)}{\lambda_{1}(t)}=\frac{\left\langle\nabla \lambda(T(t)), T^{\prime}(t)\right\rangle}{\lambda(T(t))}+\operatorname{Re} \frac{T^{\prime \prime}(t)}{T^{\prime}(t)} .
$$


From this and the assumption $v^{\prime}(x)=0$ we obtain

$$
\frac{2 x}{1-x^{2}}=\frac{\left\langle\nabla \lambda(T(x)), T^{\prime}(x)\right\rangle}{\lambda(T(x))}+\operatorname{Re} \frac{T^{\prime \prime}(x)}{T^{\prime}(x)} .
$$

Hence, by Lemma 1 and $\operatorname{Re} \frac{T^{\prime \prime}(x)}{T^{\prime}(x)}=-\frac{2 r^{2} x}{1+r^{2} x^{2}}$, we deduce that

$$
\begin{aligned}
\frac{|x|}{1-|x|^{2}} & \leq \frac{\mu|T(x)|}{1-|T(x)|^{2}}\left|T^{\prime}(x)\right|+\frac{r^{2}|x|}{1+r^{2} x^{2}} \leq \frac{\mu|T(x)|}{1-|x|^{2}}+\frac{1}{1+r^{2} x^{2}} \\
& \leq \frac{\mu}{1-|x|^{2}}+\frac{1}{1+r^{2} x^{2}},
\end{aligned}
$$

from which we obtain

$$
\frac{|x|-\mu}{1-|x|^{2}} \leq \frac{1}{1+r^{2} x^{2}}
$$

We conclude from the condition $|x|>\mu+\eta$ that

$$
\frac{\eta}{1-x^{2}}<\frac{1}{1+r^{2} x^{2}}
$$

and therefore $\frac{1+r^{2} x^{2}}{1-x^{2}}<\frac{1}{\eta}$. Finally, the equalities

$$
\frac{\left|T^{\prime}(x)\right|}{1-|T(x)|^{2}}=\frac{1}{1-|x|^{2}} \quad \text { and } \quad\left|T^{\prime}(x)\right|=\frac{1-r^{2}}{1+r^{2} x^{2}}
$$

imply

$$
\frac{1-|a|^{2}}{1-|T(x)|^{2}}=\frac{1+r^{2} x^{2}}{1-x^{2}} \leq \frac{1}{\eta}
$$

Hence and from Remark 2 and Remark 3, the assertion of the lemma follows.

The following is an immediate consequence of the lemma and (40).

Corollary 5. Under the same hypothesis as lema before, there are positive constants $C=C(\mu, \eta)$ and $\delta=\delta(\mu, \eta)$ such that

$$
\delta \leq \frac{\left(1-|y|^{2}\right) \lambda(y)}{\left(1-|a|^{2}\right) \lambda(a)} \leq C
$$

where $y=T(x)$.

Proof of Theorem 3. First observe that, as $\tilde{f}$ is bounded, there is no loss of generality in assuming that $\lambda$ has a critical point at the origin (see proof of Corollary 1 ). Under this assumption, Lemma 1 implies that

$$
\left|\frac{\partial \log \lambda}{\partial z}(z)\right| \leq \frac{\mu|z|}{1-|z|^{2}}, \quad z \in \mathbf{D} .
$$

Suppose the theorem were false. Then we could find sequences of points $\zeta_{n}^{ \pm} \in \mathbf{T}$ such that

$$
\frac{\left|\tilde{f}\left(\zeta_{n}^{+}\right)-\tilde{f}\left(\zeta_{n}^{-}\right)\right|}{\ell\left(\Gamma_{n}\right)} \leq \frac{\left|\tilde{f}\left(\zeta_{n}^{+}\right)-\tilde{f}\left(\zeta_{n}^{-}\right)\right|}{\operatorname{diam}\left(\Gamma_{n}\right)} \rightarrow 0,
$$


where $S_{n}$ is the hyperbolic segment with endpoints at $\zeta_{n}^{+}$and $\zeta_{n}^{-}$and $\Gamma_{n}=\tilde{f}\left(S_{n}\right)$. Thus, if $z_{n}$ denotes the Euclidean midpoint of $S_{n}$, from (42) and Lemma 4 one can obtain

$$
\frac{\tilde{f}\left(\zeta_{n}^{+}\right)-\tilde{f}\left(\zeta_{n}^{-}\right)}{\left(1-\left|z_{n}\right|^{2}\right) \lambda\left(z_{n}\right)} \rightarrow 0
$$

The first step of the proof consists in define a sequence of harmonic mappings $f_{n} \in$ $N H^{\mu}$ satisfying $f_{n}(0)=0$ and $s_{n}^{\prime \prime}(0)=0$, where $s_{n}$ is the arc length function of the curve $\varphi_{n}(t)=\tilde{f}_{n}(t),-1<t<1$ and $\tilde{f}_{n}$ is the lifting of $f_{n}$ with $\tilde{f}_{n}(0)=0$. For this we consider the automorphisms $T_{n}$ of the unit disk, which map $(-1,1)$ onto $S_{n}$ in such a way that $T_{n}( \pm 1)=\zeta^{ \pm}$and $T_{n}(0)=z_{n}$. Note that $T_{n}(z)=-e^{i \theta_{n}} \frac{i r_{n}+z}{1-i r_{n} z}$ if $z_{n}=r_{n} e^{i \theta_{n}}$. We have seen before that $f \circ T_{n} \in N H^{\mu}$ and $\lambda_{f \circ T_{n}}=\left(\lambda \circ T_{n}\right)\left|T_{n}^{\prime}\right|$. Proceeding as in the proof of Theorem 6 , we conclude that the functions

$$
u_{f \circ T_{n}}(t)=\frac{1}{\sqrt{\left(1-t^{2}\right) \lambda_{f \circ T_{n}}(t)}}, \quad 0<t<1
$$

have an absolute minimum at some $x_{n} \in(-1,1)$.

With $x_{n}$ as above, let $Q_{n}(z)=\frac{x_{n}-z}{1-x_{n} z}, R_{n}=T_{n} \circ Q_{n}$, and $F_{n}=f \circ R_{n}$. Note that $F_{n} \in N H^{\mu}$ and, since $Q_{n}$ fixes $(-1,1), R_{n}$ maps $(-1,1)$ onto $S_{n}$. Furthermore, $\lambda_{F_{n}}=\left(\lambda \circ R_{n}\right)\left|R_{n}^{\prime}\right|$. Hence,

$$
u_{F_{n}}(t)=\frac{1}{\sqrt{\left(1-t^{2}\right) \lambda_{F_{n}}(t)}}=\left(u_{f \circ T_{n}} \circ Q_{n}\right)(t)
$$

and consequently $u_{F_{n}}$ has a critical point at $t=0$. It follows that $\frac{\partial \lambda_{F_{n}}}{\partial x}(0)=0$. From the discussion above it is clear that the functions

$$
f_{n}=\frac{f \circ R_{n}(z)-f\left(\xi_{n}\right)}{\left.\left(1-\left|z_{n}\right|^{2}\right) \lambda_{(} z_{n}\right)}
$$

where $R_{n}(0)=T_{n}\left(x_{n}\right)=\xi_{n}$, satisfy $f_{n} \in N H^{\mu}, f_{n}(0)=0$, and $\partial_{x} \lambda_{n}(0):=\partial_{x} \lambda_{f_{n}}(0)=$ 0 . The last equality being a consequence of

$$
\lambda_{n}(z)=\frac{\lambda_{F_{n}}(z)}{\left(1-\left|z_{n}\right|^{2}\right) \lambda\left(z_{n}\right)}=\frac{\lambda\left(R_{n}(z)\right)\left|R_{n}^{\prime}(z)\right|}{\left(1-\left|z_{n}\right|^{2}\right) \lambda\left(z_{n}\right)} .
$$

We obtain also that, if $f_{n}=h_{n}+\bar{g}_{n}$, where

$$
h_{n}=\frac{h \circ R_{n}(z)-h\left(\xi_{n}\right)}{\left(1-\left|z_{n}\right|^{2}\right) \lambda\left(z_{n}\right)} \quad \text { and } \quad g_{n}=\frac{g \circ R_{n}(z)-g\left(\xi_{n}\right)}{\left(1-\left|z_{n}\right|^{2}\right) \lambda\left(z_{n}\right)}
$$

then $h_{n}(0)=g_{n}(0)=0$. Moreover, by using (41) and (44) and proceeding as in the proof of Corollary 1, we see that $\lambda_{n}$ satisfies (37) with $M=2+\mu<3$.

Next, we will show that there are positive constants $\delta$ and $C$ satisfying

$$
\delta \leq \lambda_{n}(0) \leq C \text { for } n \text { large enough. }
$$

We considerer two cases. First we suppose that there is a subsequence of $\left(x_{n}\right)$, which we will denote again by $\left(x_{n}\right)$, such that $\left|x_{n}\right|>\frac{1+\mu}{2}$ for all $n$. By defining $\eta=\frac{1-\mu}{2}$, (45) follows from Corollary 5 and the equation

$$
\lambda_{n}(0)=\frac{\left(1-\left|T_{n}\left(x_{n}\right)\right|^{2}\right) \lambda\left(T_{n}\left(x_{n}\right)\right)}{\left(1-\left|z_{n}\right|^{2}\right) \lambda\left(z_{n}\right)} .
$$


In the other case, $\left|x_{n}\right| \leq \frac{1+\mu}{2}:=K$ for all but finitely many indices $n$. Without loss of generality we may assume $\left|x_{n}\right| \leq \frac{1+\mu}{2}:=K$ for all $n$. Therefore, from

$$
\frac{\left|T_{n}^{\prime}\left(x_{n}\right)\right|}{1-\left|T_{n}\left(x_{n}\right)\right|^{2}}=\frac{1}{1-\left|x_{n}\right|^{2}} \quad \text { and } \quad\left|T_{n}^{\prime}\left(x_{n}\right)\right|=\frac{1-\left|z_{n}\right|^{2}}{1+\left|z_{n}\right|^{2} x_{n}^{2}},
$$

we have

$$
\frac{1-\left|z_{n}\right|^{2}}{1-\left|T_{n}\left(x_{n}\right)\right|^{2}}=\frac{1+\left|z_{n}\right|^{2} x_{n}^{2}}{1-\left|x_{n}\right|^{2}} \leq \frac{2}{1-K^{2}}
$$

Hence (45) follows by Remark 2 and (46).

Note that, as $\eta=\eta(\mu)$ and $K=K(\mu)$, the constants $C$ and $\delta$ in (45) depend only on $\mu$.

Without loss of generality we can assume that (45) holds for all $n$ and in virtue of Lemma 5, we can suppose that there are analytic functions $H, G$ and a positive function $\sigma$ such that $\left(h_{n}\right),\left(g_{n}\right)$, and $\left(\lambda_{n}\right)$ converge locally uniformly in $\mathbf{D}$ to $H, G$, and $\sigma$ respectively. Moreover, there are positive constants $K=K(\mu)$ and $M=M(\mu)$ which satisfy

$$
\lambda_{n}(z) \leq \frac{K}{(1-|z|)^{M}}, \quad z \in \mathbf{D} .
$$

The last steep of the proof is to show that there is a curve $\varphi \in C^{\infty}(-1,1)$ satisfying the hypothesis of Theorem 5 but is not injective in $[-1,1]$. To construct our curve, consider the sequence of lifts $\tilde{f}_{n}$ of $f_{n}$ with $\tilde{f}_{n}(0)=0$ and let $u_{n}, v_{n}, w_{n}$ be its harmonic real components. By $(47)$, the sequences $\left(u_{n}\right),\left(v_{n}\right)$ and $\left(w_{n}\right)$ are locally bounded in D. Therefore, there are harmonic functions $u, v$, and $w$ and subsequences $\left(u_{n_{k}}\right),\left(v_{n_{k}}\right)$, and $\left(w_{n_{k}}\right)$ which converge locally uniformly in $\mathbf{D}$ to $u, v$, and $w$ respectively. Consequently, the sequence of curves $\varphi_{n_{k}}(t)=\tilde{f}_{n_{k}}(t),-1 \leq t \leq 1$ converges uniformly in each compact subset of $(-1,1)$ to the curve $\varphi(t)=(u(t), v(t), w(t))$. Note that $\varphi$ is infinitely differentiable because $u, v$ and $w$ are harmonic functions in $\mathbf{D}$. Moreover, $\left|\varphi^{\prime}(t)\right|=\sigma(t) \neq 0$ for all $t \in(-1,1)$. We will prove that the convergence is uniform in $[-1,1]$. First we note that from (13) and the inequality

$$
S_{1} \varphi_{n}(t)=S s_{n}(t)+\left|\varphi_{n}^{\prime}(t)\right|^{2} k_{n}^{2}(t) \leq \frac{2 \mu}{\left(1-t^{2}\right)^{2}}, \quad-1<t<1,
$$

(see proof of Theorem 6) where $s_{n}$ is the arc length function of $\varphi_{n}$ and $k_{n}$ its curvature, it follows that

$$
S_{1} \varphi(t) \leq \frac{2 \mu}{\left(1-t^{2}\right)^{2}}, \quad-1<t<1
$$

and

$$
S s_{n}(t) \leq \frac{2 \mu}{\left(1-t^{2}\right)^{2}}, \quad-1<t<1 .
$$

Hence, since $s_{n}^{\prime \prime}(0)=\partial_{x} \lambda_{n}(0)=0, s_{n}$ satisfies (24). Thus,

$$
\frac{s_{n}^{\prime \prime}(t)}{s_{n}^{\prime}(t)} \leq \mu \frac{2 t}{1-t^{2}}, \quad 0 \leq t<1
$$

From this, by (45), one obtains by integration that

$$
\lambda_{n}(t)=s_{n}^{\prime}(t) \leq \frac{C}{\left(1-t^{2}\right)^{\mu}}, \quad 0 \leq t<1 .
$$


Now, by applying this same argument to the function $\tilde{s}_{n}(t)=-s_{n}(-t),-1<t<1$, we conclude that the inequality above also holds for $-1<t<0$. This is consequence of the fact that $\tilde{s}_{n}$ and $s_{n}$ have the same Schwarzian derivative and $\tilde{s}_{n}^{\prime \prime}(0)=s^{\prime \prime}(0)=0$.

Finally, in view of the condition $0<\mu<1$, the right member of the inequality above is integrable on $(-1,1)$. So, the sequence $\left(\varphi_{n}\right)$ is equicontinuous and bounded in $[-1,1]$. In particular, so is $\left(\varphi_{n_{k}}\right)$. Thus, each subsequence has a subsequence which converge uniformly in $[-1,1]$. It follows that $\left(\varphi_{n_{k}}\right)$ converges to $\varphi$ uniformly in $[-1,1]$ and, in consequence,

$$
\left|\varphi_{n_{k}}(-1)-\varphi_{n_{k}}(1)\right| \rightarrow|\varphi(-1)-\varphi(1)| .
$$

But, on the other hand, by definition of $\tilde{f}_{n}$,

$$
\left|\varphi_{n_{k}}(-1)-\varphi_{n_{k}}(1)\right|=\left|\tilde{f}_{n_{k}}(-1)-\tilde{f}_{n_{k}}(1)\right|=\frac{\tilde{f}\left(\zeta_{n_{k}}^{+}\right)-\tilde{f}\left(\zeta_{n_{k}}^{-}\right)}{\left(1-\left|z_{n_{k}}\right|^{2}\right) \lambda\left(z_{n_{k}}\right)},
$$

whence by (43), $\varphi(1)=\varphi(-1)$. Thus, the curve $\varphi \in C^{\infty}(-1,1)$ is not injective in $[-1,1]$, has finite length, and satisfies

$$
S_{1} \varphi(t) \leq \frac{2 \mu}{\left(1-t^{2}\right)^{2}}, \quad-1<t<1,
$$

which contradicts Theorem 5 . This contradiction completes the proof.

Acknowledgement. I wish to thank the referee for his careful reading of the previous version of this paper and his helpful suggestions, which improved the presentation of this paper.

\section{References}

[1] Ahlfors, L. V.: Cross-ratios and Schwarzian derivatives in $\mathbf{R}^{n}$. - In: Complex analysis, Birkhäuser, Basel, 1988, 1-15.

[2] Ahlfors, L. V., and G. Weill: A uniqueness theorem for Beltrami equations. - Proc. Amer. Math. Soc. 13, 1962, 975-978.

[3] Chunqui, M., P. Duren, and B. Osgood: The Schwarzian derivative for harmonic mappings. - J. Anal. Math. 91, 2003, 329-351.

[4] Chunqui, M., P. Duren, and B. Osgood: Univalence criteria for lifts of harmonic mappings to minimal surfaces. - J. Geom. Anal. 17:1, 2007, 49-74.

[5] Chunqui, M., P. Duren, and B. Osgood: Schwarzian derivative criteria for valence of analytic and harmonic mappings. - Math. Proc. Cambridge Philos. Soc. 143, 2007, 473-486.

[6] Chunqui, M., P. Duren, and B. Osgood: Two-point distortion theorems for harmonic mappings. - Illinois J. Math. 53:4, 2009, 1061-1075.

[7] Chunqui, M., P. Duren, and B. Osgood: Ahlfors-Weill extensions for a class of minimal surfaces. - In preparation.

[8] Chunqui, M, and J. Gevirtz: Simple curves in $\mathbf{R}^{n}$ and Ahlfors' Schwarzian derivative. Proc. Amer. Math. Soc. 132, 2004, 223-230.

[9] Chunqui, M., and B. Osgood: Ahlfors-Weill extensions of conformal mappings and critical points of the Poincaré metric. - Comment. Math. Helv. 69, 1994, 659-668.

[10] Chunqui, M., B. Osgood, and Ch. Pommerenke: John domains, quasidisks, and the Nehari class. - J. Reine Angew. Math. 471, 1996, 77-114.

[11] Duren, P.: Harmonic mappings in the plane. - Cambridge Univ. Press, Cambridge, U.K., 2004. 
[12] John, F.: Rotation and strain. - Comm. Pure Appl. Math. 14, 1961, 391-413.

[13] Nehari, Z.: Some criteria of univalence. - Proc. Amer. Math. Soc. 5, 1954, 700-704.

[14] Pommerenke, Ch.: Boundary behaviour of conformal maps. - Springer-Verlag, Berlin, 1992.

[15] Pommerenke, Ch., and F. W. Gehring: On the Nehari univalence criterion and quasicircles.

- Comment. Math. Helv. 59, 1984, 226-242.

Received 18 June 2012 • Accepted 19 February 2013 